\title{
231313
}

UCRL-JC-127199

PREPRINT

\section{Application of Simple Ramsauer Model to Neutron Total Cross Sections}

\author{
R. W. Bauer \\ J. D. Anderson \\ S. M. Grimes \\ V. A. Madsen
}

This paper was prepared for submittal to the

International Conference on Nuclear Data for Science and Technology

Trieste, Italy

May 19-21, 1997

April 29, 1997

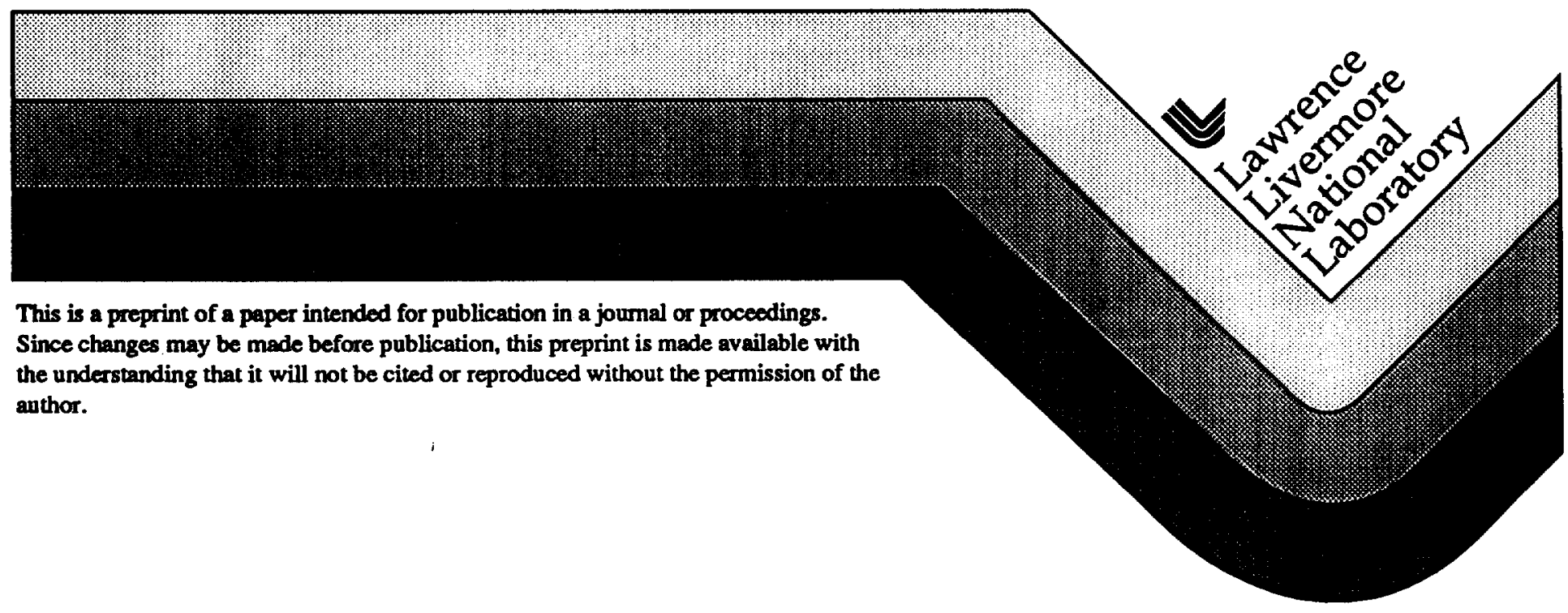




\section{DISCLAIMER}

This document was prepared as an wcoount of work sponsored by an agency of the United States Government. Neither the United States Government nor the University of California nor any of their employees, makes any warranty, express or implied, or assumes any legal liablity or responsibility for the accuracy. completeness, or usefulness of any information, apparatus, product, or process disclosed, or represents that its use would not infringe privately owned rights. Reference herein to any spectic commerdal product, process, or service by trade name, trademark, manufacture, or otherwise, does not necessarily constitute or imply its endorsement, recommendation, or favoring by the United States Government or the University of Callfornia. The views and opinions of authors expressed herein do not necessarily state or reflect those of the United States Government or the University of Callfornia, and shall not be used for advertising or product endorsement purposes. 


\title{
APPLICATION OF A SIMPLE RAMSAUER MODEL TO NEUTRON TOTAL CROSS SECTIONS
}

\author{
R.W.BAUER ${ }^{1}$, J.D.ANDERSON ${ }^{1}$, S.M.GRIMES ${ }^{2}$, V.A.MADSEN ${ }^{3}$ \\ ${ }^{1}$ Law rence Livermore National Laboratory, Livermore, CA, USA, \\ ${ }^{2}$ Ohio University, Athens, OH, USA, \\ ${ }^{3}$ Oregon State University, Corvallis, OR, USA
}

\begin{abstract}
The simple nuclear Ramsauer model has been used successfully to fit neutron total cross sections for over three decades, but has not been widely used because the foundations of the model seem so unrealistic. We have shown that the Glauber calculations with the inclusion of refraction and optical model calculations essentially validate this simple model for neutron total cross sections in the neutron energy range of $5-50 \mathrm{MeV}$. This model yields a simple formula for parameterizing the energy dependence of the neutron total cross section. We have applied the model to nuclei ranging from vanadium to bismuth. Using the simplest parameterization, we obtain fits of the data to within $2.5 \%$. With the addition of a single parameter, we can improve these fits to less than $1.5 \%$.
\end{abstract}

\section{Introduction}

The original Ramsauer analysis of scattering cross sections was applied to electron scattering from atoms at energies so low that only a single phase shift, i.e., that for $\ell=0$, was nonzero. More recently, Lawson ${ }^{1}$ and Peterson ${ }^{2}$ have applied the model to total neutron cross sections at $\mathrm{MeV}$ energies. In this situation, clearly a number of phase shifts are non-zero. If the condition for maximum cross section $(\delta=\pi / 2)$ is met at different energies for different $\ell$ values, the energy oscillations in the total cross section will be damped out. Furthermore, the energy modulations are very sensitive to the magnitude of the imaginary potential. A large value for the absorptive potential will damp out the oscillations. This damping out, for example, appears to occur for the absorption cross section for alpha particles.

The early analysis by Peterson ${ }^{2}$ showed that maxima and minima in the total cross section having the features of the Ramsauer effect could be observed. Peterson, however, did not examine the features of the nuclear potential required to produce this behavior. Franco ${ }^{3}$ used the Glauber approximation to model the process and determined that the equal phase shift assumption was not required. It was only necessary that the average phase shift vary slowly with energy for Ramsauer maxima and minima to occur. Since he did not include refraction at the nuclear surface, his results did not provide a detailed description of the data. 


\section{Analysis}

A more recent analysis by Anderson and Grimes ${ }^{4}$ examined the effects of including an isospin dependent interaction. Precision total cross sections were fit to a few percent. A paper is currently being prepared for submission to Nuclear Science and Engineering ${ }^{5}$ which examines the effectiveness of a Glauber model including refraction. It is concluded that over a significant energy range from 5 to $50 \mathrm{MeV}$, the chord length seen by a neutron traversing a nucleus is about twice the radius due to refraction. Thus we conclude that the total cross section should be well represented by

$$
\sigma_{\mathrm{T}}=2 \pi(R+\chi)^{2}(1-\alpha \cos \beta) \text {. }
$$

$R$ is the radius of the nuclear potential, $\chi$ is the reduced wave length, $\alpha$ is a parameter which is 1 if there is no absorption and is less than 1 if absorption occurs, and $\beta$ denotes the phase change in passing through the nucleus. Clearly, the "black nucleus" limit corresponds to $\alpha=0$, in which case the total cross section is $2 \pi(R+x)^{2}$. For smaller absorption, the $\alpha$ parameter will multiply the $\cos \beta$ term and allow oscillations to occur.

Finlay and collaborators ${ }^{6}$ have reported total neutron cross sections of a number of elements from 5 to $500 \mathrm{MeV}$. The following fits are based on these data. The data were first normalized by dividing the total cross section by $2 \pi(R+\chi)^{2}$ where $R=r_{0} A^{1 / 3}$ and $r_{0}$ was 1.37 as compared to 1.35 as found by Peterson ${ }^{2}$. If $\beta$ reflects the phase difference between transmitted and outside waves, it would have the form of a constant multiplying the difference $\sqrt{E+V_{0}}-\sqrt{E}$, where the constant included the radius of the nucleus. $V_{0}$ is the depth of the nuclear potential, and $\mathrm{E}$ is the kinetic energy of the neutron outside the nucleus. We, therefore, initially tried fits with $\beta$ expressed in the form:

$$
\beta=K\{\sqrt{a+b E}-\sqrt{E}\}
$$

Using this form and parameters from standard optical models ${ }^{7}$ we obtain agreement with the experimental data at about the $2.5 \%$ level. Somewhat better fits are obtained with the form:

$$
\beta=k\left\{(\sqrt{a+b \cdot E}-\sqrt{E})+k_{1}(\sqrt{a+b \cdot E}-\sqrt{E})^{2}\right\}
$$

Values for $k, a, b$, and $k_{1}$ were obtained by fitting this form to the lead total cross section values. $\alpha$ is then determined by finding how far above and below $2 \pi(R+\bar{x})^{2}$ the data go (typically $\alpha=0.11$ to 0.13 ). Using $k_{1}=0.07, a=38$ and $b=0.85$ (obtined from the lead data), the other individual elements were fit by varying $\mathrm{k}$ only. The variation of the best fit values of $\mathrm{k}$ under these constraints as a function of $\mathrm{A}^{1 / 3}$ is shown in Fig. 1. Note the approximately linear variation between these quantities as expected. In Fig. 2, we show comparisons of the reduced cross section $\sigma_{\mathrm{T}} / 2 \pi(\mathrm{R}+\chi)^{2}$ with the fit provided by Eq. (1) with $\beta$ expressed as in the form of Eq. (3). Fig $2 a$ shows the fit for the element tin, and Fig. $2 b$ the fit for lead. In each case only $\mathrm{k}$ was varied, but in these cases and in other elements measured by Finlay et $\mathrm{al}^{6}$, the $\mathrm{k}$ values fall on a straight line relative to $\mathrm{A}^{1 / 3}$ within $1.5 \%$.

\section{Summary}

The recent analysis supporting the use of Ramsauer models in parameterizing neutron total cross sections has been buttressed by fitting experimental data over a wide range of elements directly. The expression used, however, does not have any parameters reflecting deformation, closed shells or isospin. Although each of these characteristics undoubtedly influences neutron total cross sections, fits to the experimental data are achieved at the $1.5 \%$ level. This form should, therefore, be useful in estimating cross sections for targets where experimental data are unavailable. The principal limitations of these fits is that they apply 
only to energies above $6 \mathrm{MeV}$. The treatment of dispersion theory corrections will be needed to represent data at these low energies.

This work was performed under auspices of the U.S. Department of Energy by the Lawrence Livermore National Laboratory under Contract No. W-7405-ENG-48. It was also supported at Ohio University by the U.S. Department of Energy Contract No. DE-FG02-88-ER40387. The authors wish to thank F.S. Dietrich for providing unpublished total cross section data.

References:

1. J.D. Lawson, Phil. Mag. 44, 102 (1953)

2. J.M. Peterson, Phys. Rev. 125, 955 (1962)

3. V. Franco, Phys. Rev. 140, B1501 (1965)

4. J.D. Anderson and S.M. Grimes, Phys. Rev. C41, 2904 (1990)

5. S.M. Grimes, J.D. Anderson, R.W. Bauer and V.A. Madsen, to be submitted to Nucl. Sci. and Eng. (1997)

6. R.W. Finlay et al.. Phys. Rev. C 47, 237 (1993) and F.S. Dietrich, to be published

7. J. Rapaport, Physics Reports 87, 25 (1982)

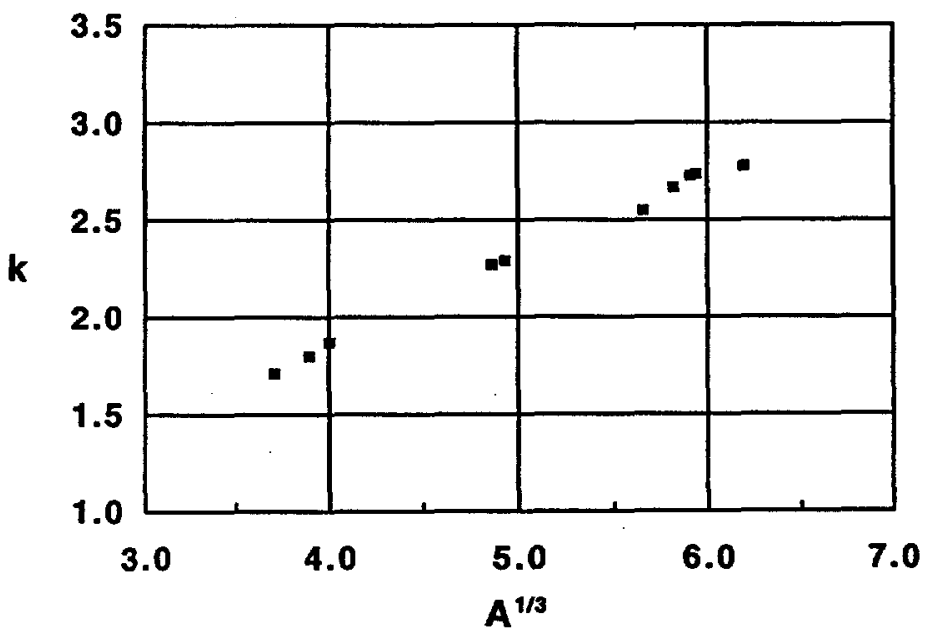

Fig. 1. The variation of the best fit values of parameter $k$ from Eq. (3), keeping $k_{1}$, $a$ and $b$ constant, as a function of $A^{1 / 3}$, ranging from vanadium to uranium.

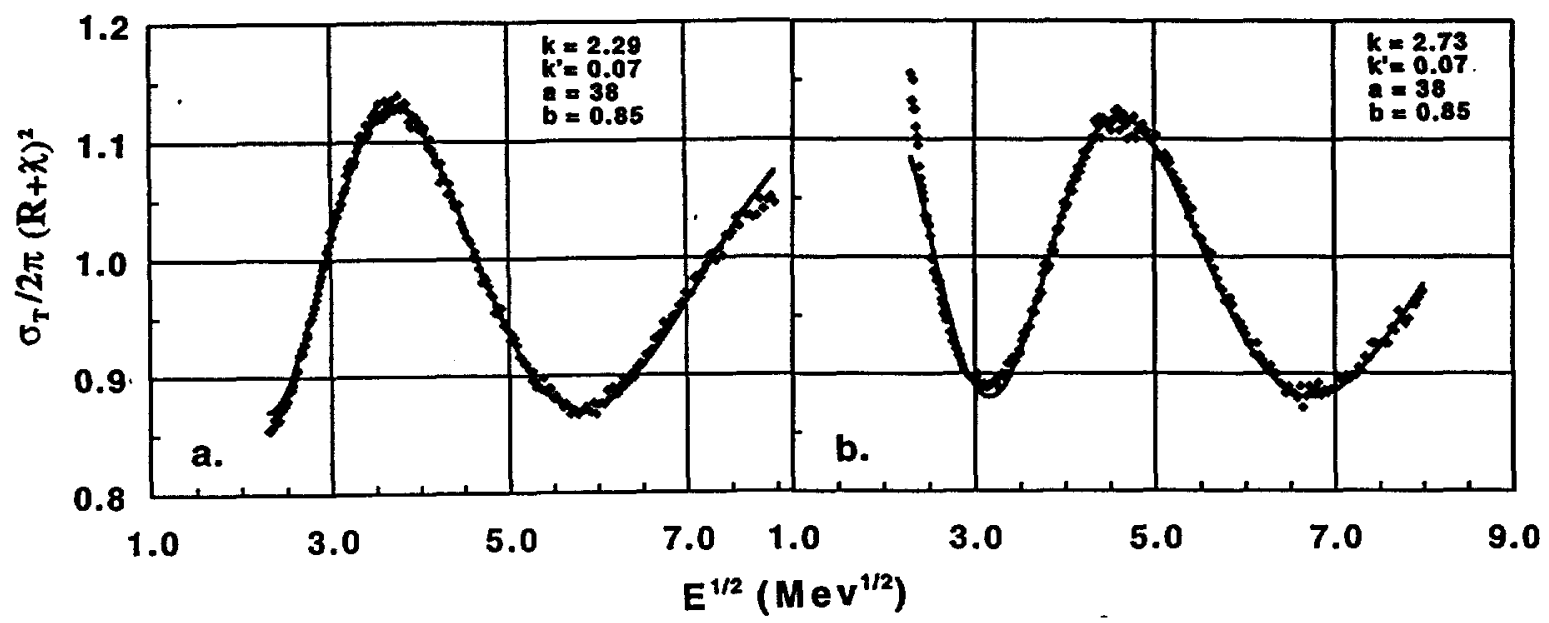

Fig. 2. Comparisons of the reduced cross section $\sigma_{\mathrm{T}} / 2 \pi(\mathrm{R}+\chi)^{2}$ with the fit provided by $\mathrm{Eq}$. (1) with $\beta$ expressed as in the form of Eq. (3). Fig $2 \mathrm{a}$ shows the fit for tin, Fig. $2 \mathrm{~b}$ for lead. 


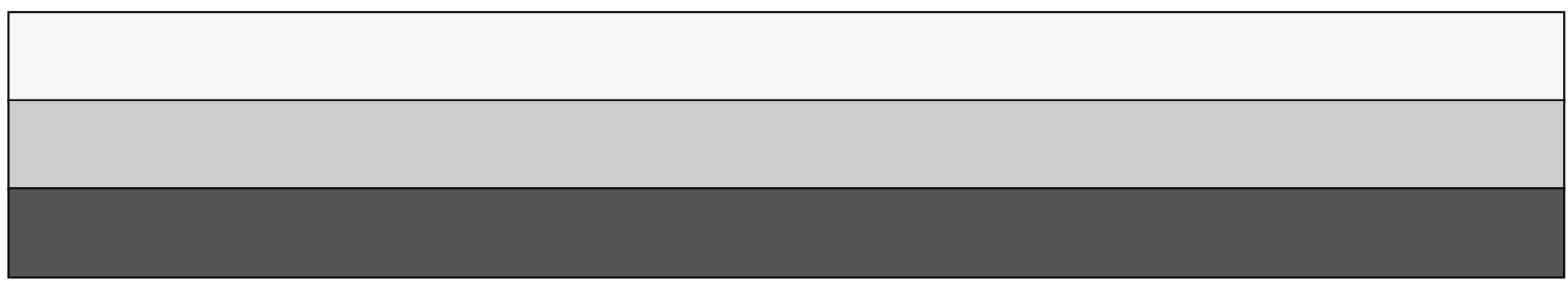

